\title{
Epidemiology of dentin hypersensitivity
}

\author{
Christian H. Splieth • Aikaterini Tachou
}

Received: 2 December 2011 / Accepted: 21 November 2012 /Published online: 7 December 2012

(C) Springer-Verlag Berlin Heidelberg 2012

\begin{abstract}
Objective In contrast to the well-established caries epidemiology, data on dentin hypersensitivity seem to be scarce and contradictory. This review evaluates the available literature on dentin hypersensitivity and assesses its prevalence, distribution, and potential changes.

Materials and methods The systematic search was performed to identify and select relevant publications with several key words in electronic databases. In addition, the articles' bibliographies were consulted.

Results Prevalence rates range from 3 to $98 \%$. This vast range can be explained partly by the differences in the selection criteria for the study sample and also the variety in diagnostic approaches or time frames. Women are slightly more affected than men and an age peak of 30-40 years has been reported. Still, the prevalence of erosions with dentin exposure seems to increase in younger adults, often resulting in hypersensitivity. In older patients, root surfaces are frequently exposed due to periodontal disease which is associated with a high rate of dentin hypersensitivity, especially after periodontal treatment and intensified brushing activity. On the other hand, the number of affected seniors with tooth loss or even edentulism is reduced. About 25 $30 \%$ of the adult population report dentin hypersensitivity. Most dentists also consider it to be a relevant problem in their practice, but they request more information on this topic. Maxillary teeth are affected to a higher extent, but the different teeth show very similar rates. Buccal surfaces clearly show the highest prevalence rates.
\end{abstract}

C. H. Splieth $(\bowtie) \cdot$ A. Tachou

Department of Preventive and Pediatric Dentistry,

Center for Oral Health, Ernst Moritz Arndt University Greifswald,

Rotgerberstraße 8,

17487 Greifswald, Germany

e-mail: splieth@uni-greifswald.de
Conclusion In spite of the advances regarding management of dentin hypersensitivity, it still remains an epidemiologically understudied field.

Clinical relevance Although great variations have been observed in the prevalence of dentin hypersensitivity, this issue is often observed by dentists and related by patients. However, further studies are necessary to find the cause of this condition and refine its management.

Keywords Epidemiology $\cdot$ Prevalence $\cdot$ Dentin(e) hypersensitivity $\cdot$ Age $\cdot$ Gender

\section{Introduction}

In 1987, Locker and Grushka analyzed the impact of dental and facial pain in a representative Canadian sample. It was one of the first studies that tried to assess dental pain and its social or psychological impact systematically. Overall, $39.7 \%$ of the respondents reported dental, oral, or facial pain within the last 4 weeks including $28.8 \%$ "pain in teeth with hot and cold fluids" as the most prevalent oral pain. The majority of dental and facial pain had a psychological or behavioral impact (58 and $70 \%$, resp.). Thus, dentofacial pain is a relevant problem which is mostly associated with tooth sensitivity [1]. Apart from carious destruction resulting in pulpitis or postoperative hypersensitivity, dental pain can be ascribed to dentin hypersensitivity (DHS) which is characterized by short, sharp pain originating from exposed dentin in response to stimuli, typically thermal, evaporative, tactile, osmotic, or chemical, and which cannot be ascribed to any other form of dental defect or pathology [2].

In contrast to the well-established research in caries epidemiology, data on dentin hypersensitivity are scarce and vary considerably. Prevalence rates range from 3 to $98 \%$, a heterogeneity which can be explained by the difference in the selection criteria used for each study sample and also in 
the diagnostic approaches $[3,4]$. Therefore, the aim of this review is to analyze the available literature on dentin hypersensitivity and to assess its prevalence, distribution, perception by dentists, and its potential future development.

\section{Materials and methods}

A systematic search was performed to identify and select relevant studies. The key words used in the searches were "dentin(e)" and "hypersensitivity," "caries" and combinations of them with "prevalence," "longitudinal," "epidemiology," "remission," "review," and "treatment", respectively.

The electronic databases searched included PubMed, Medline, Social Science Citation Index, and Science Citation Index Expanded. No publication time limitations were applied. Nevertheless, in an attempt to limit the results to the most relevant ones, search in PubMed database was confined to the article titles/abstracts, published in German and English. Unfortunately, as the option of "title/abstract" was not available, Medline and Science Citation Index as well as the Science citation index expanded searches were limited to the article titles. The fulltext copies of the relevant publications were obtained and read. The article bibliographies were also consulted.

\section{Results}

The fact that publications on DHS were totally outnumbered by caries-relevant ones was evident. The great difference in numbers, as can be clearly noted in Table 1 , could be observed in all the searches that were performed. In addition, there was a vast variety of approaches including studies on postoperative hypersensitivity following adhesive restorations, bleaching $[5,6]$, or periodontal treatment $[7,8]$. Out of all caries-related search fields, prevalence scored the greatest number of hits in all databases. Prevalence and epidemiology of dentin hypersensitivity, however, proved to be rather understudied, as the number of relevant publications was substantially smaller, thereby exhibiting the need for further research on this field.

Many heterogeneous study populations were examined for DHS. Nevertheless, with the exception of one study on global prevalence [9] and one national Chinese study [10], no nationally representative sample was drawn.

As far as DHS epidemiology was concerned, available data were scarce, and out of the three studies which met the search criteria, two were more treatment-related than actually focused on epidemiological data $[11,12]$. Furthermore, few studies exist on the remission of dental hypersensitivity as well as for its evaluation methods, and, whereas there seems to be an abundance of longitudinal studies about caries, only nine relevant search results were found for DHS. On the contrary, it seemed that treatment was the main domain where dentin hypersensitivity research has focused till the present day, as was clearly demonstrated by the large number and variety of related publications.

\section{Prevalence and distribution}

As in early caries epidemiology, the literature on dentin hypersensitivity presents various puzzle pieces, and different

Table 1 Results for literature search with keywords "dentin(e)“ and "hypersensitivity" plus additional keywords and respective number of hits for "caries" plus additional keywords

\begin{tabular}{|c|c|c|c|c|}
\hline & PUBMED & MEDLINE & SciSearch and Social SciSearch & Comments and observations \\
\hline Caries & 22,209 & 12,625 & 10,274 & \multirow[t]{2}{*}{ Huge difference in the number of available studies } \\
\hline DHS & 451 & 227 & 185 & \\
\hline Caries and prevalence & 858 & 858 & 888 & \multirow{2}{*}{$\begin{array}{l}\text { Considerably fewer studies for DHS than caries. V ast variety } \\
\text { of populations examined. With the exception of } 1 \text { study on } \\
\text { global prevalence, no nationally representative sample was } \\
\text { drawn for DHS. }\end{array}$} \\
\hline DHS and prevalence & 36 & 6 & 11 & \\
\hline Caries and epidemiology & 68 & 68 & 45 & \multirow{2}{*}{$\begin{array}{l}\text { Astonishing lack of relevant DHS studies. Out of the } 3 \\
\text { available, } 2 \text { are treatment-related. }\end{array}$} \\
\hline DHS and epidemiology & 3 & 0 & 1 & \\
\hline Caries and longitudinal & 615 & 134 & 155 & \multirow{2}{*}{$\begin{array}{l}\text { Abundance of longitudinal data available for caries. Only } \\
\text { few and management-related longitudinal studies for DHS. }\end{array}$} \\
\hline DHS and longitudinal & 9 & 0 & 0 & \\
\hline Caries and remission & 10 & 1 & 1 & \multirow{2}{*}{$\begin{array}{l}\text { Caries hits are partly associated with remission of other } \\
\text { general diseases. Too few data available for DHS remission } \\
\text { and its evaluation methods }\end{array}$} \\
\hline DHS and remission & 2 & 0 & 0 & \\
\hline Caries and review & 1,121 & 216 & 106 & \multirow{2}{*}{$\begin{array}{l}\text { Many of the DHS reviews are actually only reviews of the } \\
\text { various management methods available. }\end{array}$} \\
\hline DHS and review & 43 & 13 & 8 & \\
\hline Caries and treatment & 4,095 & 457 & 374 & \multirow[t]{2}{*}{ Great variety of treatment methods for DHS } \\
\hline DHS and treatment & 279 & 56 & 47 & \\
\hline
\end{tabular}

DHS dentin hypersensitivity 
studies seem to be partly contradictory. Cross-sectional surveys in dental offices which reveal prevalence rates of 3-5\% seem to indicate that dentin hypersensitivity might be a minor or even irrelevant problem $[13,14]$. On the other hand, with a prevalence of $84.5 \%$, almost all patients experience hypersensitivity after periodontal treatment [8] and most practitioners in a UK sample $(72 \%)$ stated that dentin hypersensitivity is a severe problem for at least $10 \%$ of their patients [15]. In addition, they expressed a need for appropriate scientific information on prevention and treatment.

Most review articles conclude that the prevalence of dentin hypersensitivity ranges from 3 to $57 \%[16,17]$ depending on the study sample. Regarding gender, women tend to be more frequently affected than men, although these differences often do not reach a statistically significant level [18-22].

Early research found that all age groups are affected [22], but also an age peak from 30 to 40 years has been reported $[18,21,23]$. A reduction in seniors could be plausible due to the development of secondary or reparative dentin and subsequent sclerosis of the dentinal tubules accompanied by reduced sensitivity. In contrast to this, prevalence rates from 22 to $30 \%$ in adults without clear age pattern were found in a representative Canadian sample [17].

Regarding the intraoral distribution, dentin hypersensitivity is mostly associated with exposed buccal dentin in permanent teeth [18, 24]. Addy and Pearce [25] connected that to vigorous tooth brushing. This gives a clear hint to an etiological factor for dentin hypersensitivity and was also confirmed by a recent review [26]. Supposedly, the left side is more affected due to mostly right-handed brushing.

Other potential causes of dentin hypersensitivity are erosion, gingival recessions, or abfractions per se or in combination with vigorous tooth brushing. They are thought to result in exposed cervical dentin, open dentin tubules, and painful sensations to thermal, tactile, or other stimuli [24, 27-30]. Thus, the etiological factors clearly explain the intraoral distribution with a high preference for the buccal surfaces.

In general, maxillary teeth seem to be more affected than the mandibular [25], but this often fails to reach a statistically significant level [18]. The distribution among teeth favors premolars, for instance, in Gillam et al. [31] with $30.6 \%$ being affected compared to canines with only $14.9 \%$. On the other hand, there is only one canine for two premolars, and dividing the premolar rate by two gives almost identical values for premolars and canines. The very detailed recording in a recent Australian study reveals that the first and second premolars, the first molars, and canines are all affected at very similar rates ranging from 8 to $11 \%$ in the maxilla and 4 to $9 \%$ in the mandible [18]. This is also plausible, when erosion, abfractions, and/or vigorous tooth brushing are considered as etiological factors $[27,28]$. As a direct result, these teeth are all in line and exposed to similar conditions.

\section{Professionals' view}

In a survey, UK dentists were asked about their perception and knowledge on dentin hypersensitivity [15]. They stated that about $25 \%$ of their patients suffered from dentin hypersensitivity. Furthermore, the vast majority of dentists (72\%) considered it to be a severe problem in at least $10 \%$ of their patients. In spite of various treatment approaches such as careful brushing instructions, desensitizing toothpaste, fluoride applications, or dentin bonding agents, the dentists highlighted that certain aspects of this topic were lacking such as appropriate scientific information including prevention and treatment.

These uncertainties among dentists were also detected in a Canadian survey where fewer than half of them considered a differential diagnosis, although dentin hypersensitivity is, by definition, a diagnosis of exclusion [17]. The majority of the dentists and dental hygienists identified bruxism and malocclusion as triggers of dentin hypersensitivity even though neither has been identified as a major causative factor. In addition, $17 \%$ of the dentists and $48 \%$ of dental hygienists failed to identify the hydrodynamic theory as underlying mechanism. In conclusion, dentin hypersensitivity is a relevant problem for patients and dentists, but scientifically and in dental education, it has not been examined to the same extent as other oral problems.

In a very recent study, Australian dentists were a little bit more relaxed about dentin hypersensitivity: Most dentists rated the occurrence below $20 \%$, and the overwhelming majority clearly identified the underlying pathological mechanism, potential etiological factors as well as the appropriate hierarchy of treatment options [32].

\section{Where are the problems?}

For an epidemiologist, it is puzzling to find a range in prevalence studies from 3 to $98 \%$ in one country for the same disorder [14, 33]. Apart from real differences, a possible explanation lies in various biases. The most obvious one is a selection bias in the recruitment of the study sample. In contrast to many caries studies with representatively selected samples, most studies on dentin hypersensitivity are restricted to highly selected populations such as patients at periodontal offices [8, 33], students [28], or hospitalized patients [34]. This explains already a lot about the variation in prevalence rate. But also "representative" studies concerning patients in general practice vary considerably. First of all, the pattern in general dentistry can also differ: In some countries and practices, the focus is on prevention, and most patients turn up for regular recall visits. In other settings, patients are mostly scheduled for restorative or periodontal treatment which shifts the study sample towards higher disease categories. This was confirmed by a national representative survey on oral health in 
Germany [35] where the DMFT data in adults were slightly lower than the data from a national survey in patients from dental offices in the same year [36]. In addition, it is questionable if individuals with dentin hypersensitivity automatically seek professional help, especially when dentists themselves are lacking appropriate scientific information including prevention and treatment of hypersensitivity [15].

There is also conflicting evidence for the gender distribution in dentin hypersensitivity: The numbers of affected females tend to be higher than those of males, though often failing to reach the level of statistical significance $[8,18]$. A possible explanation could be that women tend to brush more intensively than men $[37,38]$ and that they eat more healthy fruity food items which are also erosive [39]. This combination of erosion and abrasion presents an ideal mixture of etiological risk factors for dentin hypersensitivity. Furthermore, women tend to visit the dentist more frequently [40] which results in an overrepresentation and, therefore, classical selection bias if the study sample is recruited in dental practices. In addition, women articulate health problems more willingly than men [41], which leads to further detection bias for women.

Another reason for the varying prevalence data could be that different diagnostic criteria are used in the studies. Even within the same study, prevalences differ when tactile or thermal tests are applied [42]. Thus, it is very likely that clinical tests [31], self-reporting by patients [13, 43], or the dentists' assessment [15] result in different prevalence values for the same sample.

Another interesting point of discussion is the age distribution. Almost all studies report a reduction in prevalence for seniors, but also the number of dentate individuals, who exhibit teeth at risk, decreases with age. A similar epidemiological problem can be observed in root caries (Fig. 1). With tooth loss and edentulism, the number of teeth and individuals at risk is constantly reduced in a "classical" population. The distribution of exposed cervical dentin in Fig. 1 reflects the age distribution of dentin hypersensitivity

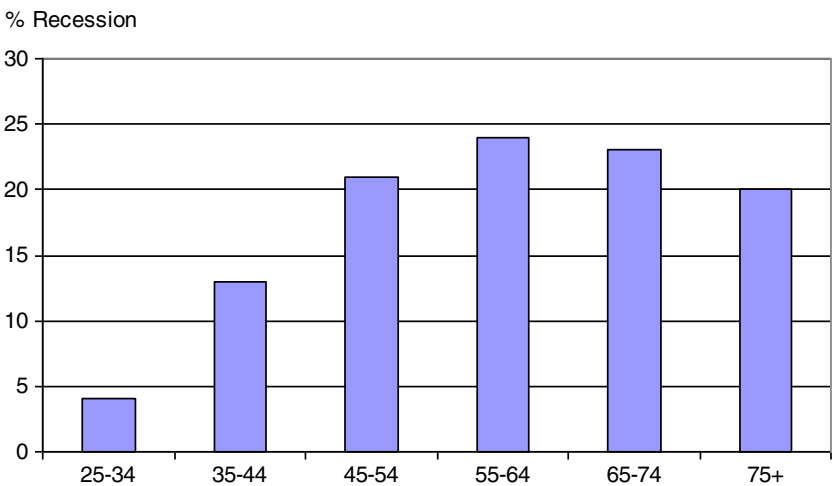

Fig. 1 Prevalence of exposed root surfaces, gingival recessions, and, therefore, exposed cervical dentin in German adults [30] assessed in Australian dental offices [18]. Therefore, it is important to calculate the relative risk for root caries which increases with age, meaning that the proportion of retained teeth affected by root caries grows continuously [29].

As far as dentin hypersensitivity is concerned, the relative risk could be constant, and the clear peak that is observed in patients around 40 years of age presenting with dentin hypersensitivity in dental practices might be explained by the high rate of exposed root surfaces at that age. In seniors, the lower number could simply be based on the reduced number of teeth present. In the near future, this question will be of growing importance, as the number of retained teeth increases in adults. This means a marked increase in the number of teeth and individuals at risk for exposed cervical dentin and dentin hypersensitivity will be observed in the senior population. The rise will be especially prominent if it is accompanied by improved and prolonged toothbrushing and/or erosive food components which are considered as major etiological risk factors for dentin hypersensitivity [27, $28,44]$. In countries with a long-standing history of prevention and, therefore, a high number of dentate seniors, such as Canada [45], the prevalence of dentin hypersensitivity is independent of age, at a rate of about $25-30 \%$ [17].

\section{Development over time}

Longitudinal epidemiological studies on dentin hypersensitivity hardly exist at all, but several clinical studies examine development over a few weeks, mostly in the context of the effectiveness of desensitizing agents or following dental treatment. Hypersensitivity due to procedures such as adhesive restorations, periodontal surgery, or bleaching has been examined under the term of "sensitivity," which does not exactly reflect the situation as far as exposed cervical dentin is concerned [46]. Still, $54 \%$ of the randomly selected control group in a study on bleaching reported mild sensitivity which decreased to $6 \%$ after 4 weeks using a placebo gel [47]. In addition, the presence of gingival recessions increased the probability of tooth (hyper)sensitivity significantly, but this also decreased as the treatment progressed.

As mentioned above, periodontal treatment almost automatically leads to dentin hypersensitivity [48]. In a recent American study [42], the prevalence of $30 \%$ preoperative tactile and thermal hypersensitivity increased to 67 and $76 \% 1$ week after periodontal surgery, depending on the diagnostic stimulus used. Including the subjective patient statement, the combined prevalence even reached $79 \%$. After 6 weeks, dentin hypersensitivity had decreased dramatically to almost preoperative values, even-as the authors state-without the use of desensitizing therapy. Thus, iatrogenic dentin hypersensitivity exhibits a high rate of spontaneous remission without professional treatment. There are probably some patients who apply desensitizing 
toothpastes on their own accord [49], but it can be speculated that dentin hypersensitivity shows the same fluctuating patterns as other kinds of somatic pain [50]. This is most likely, as in randomized clinical trials, even patients of the placebo control group experience a remission of their dentin hypersensitivity, although the reduction has been more pronounced in the test group with the therapeutic dentifrice [51-53].

\section{Future research implications}

In order to assess the true prevalence of dentin hypersensitivity, a study with a population-based representative sample is needed. As dentin hypersensitivity per se is not considered a serious problem, this can be done most likely as an add-on in other health surveys - or as a questionnaire study. In addition, the researchers should be aware of the concept in pain research [50], where 1-month, 3-month, or 1-year prevalence rates are assessed. The results will possibly show that the life-time prevalence is close to $100 \%$, while the $1-$ 3 -month prevalences will range from 10 to $30 \%$ in adults with very little variation for gender or age in a population sample which is corrected for tooth loss.

Far more interesting than the exact prevalence rate is the development of dentin hypersensitivity over time and the rate of spontaneous remission or with self-treatment at home. The remission rates after iatrogenic (hyper)sensitivity due to periodontal surgery, bleaching, or restorative treatment $[42,46$, 47] are high, but the situation might be very different in hypersensitivity associated with chronic exposure to erosive food or drinks, vigorous tooth brushing, or abfractions [54] due to functional problems which persist. Control groups with placebo in trials on desensitizing agents also exhibit impressive rates of remission, but incidence rates with relapses in these patients are unknown. Thus, longitudinal data from pain diaries are needed to understand the patterns and spontaneous remission rate of dentin hypersensitivity. In addition, randomized placebo control groups are essential in any clinical trial on dentin hyperactivity as the therapeutic can only be assessed against the natural fluctuation of the condition. Holland et al. [1] also stressed the importance of double-blinding these studies to prevent an observer bias [2].

This is of special interest, as most factors indicate a potential increase in dentin hypersensitivity: The number of retained teeth rises (IDZ 2006), often associated with intensified oral hygiene which can lead to exposed cervical dentin [36]. In addition, young adults such as Swiss recruits often exhibit erosions with dentin involvement $(30.7 \%)$ or wedgeshaped defects $(20.4 \%)$ [55] which run a high risk for dentin hypersensitivity. This was also confirmed by Bamise et al. (2010) who recorded a prevalence of $68 \%$ for dentin hypersensitivity in university students, often associated with erosion and the use of a hard toothbrush [28].

\section{Conclusions/clinical relevance}

In conclusion, dentin hypersensitivity is a clinically relevant and population-wide problem. Although dentin hypersensitivity may affect about a quarter of the adult population, they often do not see the dentist as the right person to consult. It also seems that dentists themselves are not always fully informed on the etiological or diagnostic concept of dentin hypersensitivity or its treatment, which can be explained by the gaps in research. Although dentin hypersensitivity is not considered a lethal problem, it affects the quality of life of patients and, therefore, it should be properly addressed in research, dental education, prevention, and treatment.

Conflict of interest The authors state that they do not have a conflict of interest.

\section{References}

1. Locker D, Grushka M (1987) The impact of dental and facial pain. J Dent Res 66:1414-1417

2. Holland GR, Narhi MN, Addy M, Gangarosa L, Orchardson R (1997) Guidelines for the design and conduct of clinical trials on dentine hypersensitivity. J Clin Periodontol 24:808-813

3. Addy M (1990) Etiology and clinical implications of dentine hypersensitivity. Dent Clin North Am 34:503-514

4. Chabanski MB, Gillam DG (1997) Aetiology, prevalence and clinical features of cervical dentine sensitivity. J Oral Rehabil 24:15-19

5. Goldberg M, Grootveld M, Lynch E (2010) Undesirable and adverse effects of tooth-whitening products: a review. Clin Oral Investig 14:1-10

6. Haywood VB (2002) Dentine hypersensitivity: bleaching and restorative considerations for successful management. Int Dent $\mathrm{J}$ 52:376-385

7. Von Troil B, Needleman I, Sanz M (2002) A systematic review of the prevalence of root sensitivity following periodontal therapy. J Clin Periodontol 29(Suppl 3):173-177, discussion 195-6

8. Chabanski MB, Gillam DG, Bulman JS, Newman HN (1996) Prevalence of cervical dentine sensitivity in a population of patients referred to a specialist Periodontology Department. J Clin Periodontol 23:989-992

9. Graham FL, Tatton-Brown C, Meert G, Alexander DC (2002) Prevalence and severity of dentine hypersensitivity: a global perspective. J Dent Res 82(special issue B):134

10. Rong WS, Hu DY, Feng XP, Tai BJ, Zhang JC, Ruan JP (2010) A national survey on dentin hypersensitivity in Chinese urban adults. Zhonghua Kou Qiang Yi Xue Za Zhi 45:141-145

11. Cummins D (2010) Recent advances in dentin hypersensitivity: clinically proven treatments for instant and lasting sensitivity relief. Am J Dent 23(Spec No A):3A-13A

12. Cummins D (2009) Dentin hypersensitivity: from diagnosis to a breakthrough for everyday sensitivity relief. J Clin Dent 20:1-9

13. Rees JS, Addy M (2002) A cross-sectional study of dentine hypersensitivity. J Clin Periodontol 29:997-1003

14. Rees JS, Addy M (2004) A cross-sectional study of buccal cervical sensitivity in UK general dental practice and a summary review of prevalence studies. Int J Dent Hyg 2:64-69 
15. Gillam DG, Bulman JS, Eijkman MA, Newman HN (2002) Dentists' perceptions of dentine hypersensitivity and knowledge of its treatment. J Oral Rehabil 29:219-225

16. Orchardson R, Gilliam D (2006) Managing dentin hypersensitivity. J Am Dent Assoc 137:990-998

17. Canadian Advisory Board on Dentin Hypersensitivity (2003) Consensus-based recommendations for the diagnosis and management of dentin hypersensitivity. J Can Dent Assoc 69:222-226

18. Amarasena N, Spencer J, Ou Y, Brennan D (2011) Dentine hypersensitivity in a private practice patient population in Australia. $\mathrm{J}$ Oral Rehabil 38:52-60

19. Bekes K, Schaller H-G, Hirsch C (2008) Verbesserung mundgesundheitsbezogener Lebensqualität durch die Anwendung von Mundhygieneprodukten für schmerzempfindliche Zähne und freiliegende Zahnhälse. ZWR 117:75-80

20. Oyama T, Matsumoto K (1991) A clinical and morphological study of cervical hypersensitivity. J Endod 17:500-502

21. Flynn J, Galloway R, Orchardson R (1985) The incidence of hypersensitive teeth in the west of Scotland. J Dent 13:230-236

22. Fischer C, Fischer RG, Wennberg A (1992) Prevalence and distribution of cervical dentine hypersensitivity in a population in Rio de Janeiro, Brazil. J Dent 20:272-276

23. Graf H, Galasse R (1977) Morbidity, prevalence and intraoral distribution of hypersensitive teeth. J Dent Res 56(special issue a): 162

24. Dababneh R, Khouri A, Addy M (1999) Dentine hypersensitivity: an enigma? A review of terminology, epidemiology, mechanisms, aetiology and management. Br Dent J 187:606-611

25. Addy M, Pearce N (1994) Aetiological, predisposing and environmental factors in dentine hypersensitivity. Archs Oral Biol 39:3338

26. Addy M (2006) Tooth brushing, tooth wear and dentine hypersensitivity - are they associated? J Ir Dent Assoc 51:226-231

27. Bamise CT, Olusile AO, Oginni AO (2008) An analysis of etiological and predisposing factors related to dentin hypersensitivity. $\mathrm{J}$ Contemp Dent Pract 1(9):52-59

28. Bamise CT, Kolawole KA, Oloyede EO, Esan TA (2010) Tooth sensitivity experience among residential university students. Int $\mathrm{J}$ Dent Hyg 8:95-100

29. Ommerborn MA, Schneider C, Giraki M, Schafer R, Singh P, Franz M, Raab WH (2007) In vivo evaluation of noncarious cervical lesions in sleep bruxism subjects. J Prosthet Dent 98:150 158

30. Splieth C, Schwahn C, Bernhardt O, John U (2004) Prevalence and distribution of root caries in Pomerania, North-East Germany. Caries Res 38:333-340

31. Gillam DG, Aris A, Bulman JS, Newman HN, Ley F (2002) Dentine hypersensitivity in subjects recruited for clinical trials: clinical evaluation, prevalence and intra-oral distribution. J Oral Rehabil 29:226-231

32. Amarasena N, Spencer J, Ou Y, Brennan D (2010) Dentine hypersensitivity-Australian dentists' perspective. Aust Dent J 55:181187

33. Chabanski MB, Gillam DG, Bulman JS, Newman HNJ (1997) Clinical evaluation of cervical dentine sensitivity in a population of patients referred to a specialist periodontology department: a pilot study. Oral Rehabil 24:666-672

34. Rees JS, Jin U, Lam S, Kudanowska I, Vowles R (2003) The prevalence of dentine hypersensitivity in a hospital clinic population in Hong Kong. J Dent 31:453-461

35. Micheelis W, Bauch J (1996) Oral health of representative samples of Germans examined in 1989 and 1992. Community Dent Oral Epidemiol 24:62-67
36. Dünninger P, Uhl T, Einwag J, Naujoks R (1995) Die Veränderungen der Mundgesundheit in der Bundesrebublik Deutschland-das Projekt A10. Dtsch Zahnarztl Z 50:40-44

37. Gillam DG, Seo HS, Newman HN, Bulman JS (2001) Comparison of dentine hypersensitivity in selected occidental and oriental populations. J Oral Rehabil 28:20-25

38. Sakalauskienė Z, Vehkalahti MM, Murtomaa H, Mačiulskienė V (2011) Factors related to gender differences in toothbrushing among Lithuanian middle-aged university employees. Medicina (Kaunas) 47:180-186

39. Westenhoefer J (2005) Age and gender dependent profile of food choice. In: Elmadfa I (ed) Diet diversification and health promotion Forum Nutr. Karger, Basel, pp 44-51

40. Nuttall NM, Bradnock G, White D, Morris J, Nunn J (2001) Adult Dental Health Survey: dental attendance in 1998 and implications for the future. BDJ 190:177-182

41. Noone JH, Stephens C (2008) Men, masculine identities, and health care utilisatiion. Sociol Health Illn 30:711-725

42. Al-Sabbagh M, Beneduce C, Andreana S, Ciancio SG (2010) Incidence and time course of dentinal hypersensitivity after periodontal surgery. Gen Dent 58:e14-e19

43. Rees JS (2000) The prevalence of dentine hypersensitivity in general dental practice in the UK. J Clin Periodontol 27:860-865

44. Walters PA (2005) Dentinal hypersensitivity: a review. J Contemp Dent Pract 2(6):107-117

45. Lewis DW, Ismail AI (1995) The Canadian Task Force on the Periodic Health Examination 1995 update: 2. Prevention of dental caries. Can Med Assoc J 152(6)

46. Swift EJ Jr, Ritter AV, Heymann HO, Sturdevant JR, Wilder AD Jr (2008) 36-month clinical evaluation of two adhesives and microhybrid resin composites in Class I restorations. Am J Dent 21 (3):148-152

47. Jorgensen MG, Carroll WB (2002) Incidence of tooth sensitivity after home whitening treatment. J Am Dent Assoc 133:1076-1082

48. Taani Q, Awartani F (2002) Clinical evaluation of cervical dentin sensitivity (CDS) inpatients attending general dental clinics (GDC) and periodontal specialty clinics (PSC). J Clin Periodontol 29:118122

49. Irwin CR, McCusker P (1997) Prevalence of dentine hypersensitivity in general dental population. J Irish Dent Assoc 43:7-9

50. Suri P, Rainville J, Fitzmaurice GM, Katz JN, Jamison RN, Martha J, Hartigan C, Limke J, Jouve C, Hunter DJ (2011) Acute low back pain is marked by variability: an internet-based pilot study. BMC Musculoskelet Disord 5(12):220

51. Pradeep AR, Sharma A (2010) Comparison of clinical efficacy of a dentifrice containing calcium sodium phosphosilicate to a dentifrice containing potassium nitrate and to a placebo on dentinal hypersensitivity: a randomized clinical trial. J Periodontol 81:11671173

52. Du Min Q, Bian Z, Jiang H, Greenspan DC, Burwell AK, Zhong J, Tai BJ (2008) Clinical evaluation of a dentifrice containing calcium sodium phosphosilicate (novamin) for the treatment of dentin hypersensitivity. Am J Dent 21:210-214

53. Ghassemi A, Hooper W, Winston AE, Sowinski J, Bowman J, Sharma N (2009) Effectiveness of a baking soda toothpaste delivering calcium and phosphate in reducing dentinal hypersensitivity. J Clin Dent 20:203-210

54. Bernhardt O, Gesch D, Schwahn C, Mack F, Meyer G, John U, Kocher T (2006) Epidemiological evaluation of the multifactorial aetiology of abfractions. J Oral Rehabil 33:17-25

55. Jaeggi T, Schaffner M, Bürgin W, Lussi A (1999) Erosions and wedge-shaped defects in Swiss Army recruits. Schweiz Monatsschr Zahnmed 109:1170-1182 